\title{
Evolutionary status and fundamental parameters of the Cepheid V1033 Cyg
}

\author{
Yu. A. Fadeyev \\ Institute of Astronomy, Russian Academy of Sciences, Pyatnitskaya ul. 48, Moscow, 119017 \\ Russia
}

Received June 15, 2020; revised June 17, 2020; accepted June 26, 2020

\begin{abstract}
On the basis of consistent stellar evolution and nonlinear stellar pulsation calculations the Cepheid V1033 Cyg is shown to be the post-main sequence star at the first crossing of the instability strip during gravitational contraction of the helium core. The observed light variability of V1033 Cyg is due to radial oscillations in the fundamental mode. The best agreement (within one percent) between recent observations and the theoretical estimate of the period change rate was obtained for the evolutionary sequence with stellar mass $M=6.3 M_{\odot}$ and helium and heavier element fractional abundances $Y=0.28$ and $Z=0.022$, respectively. The age of the star, the luminosity, the radius, the effective temperature and the surface gravity are $t_{\mathrm{ev}}=5.84 \times 10^{7} \mathrm{yr}, L=2009 L_{\odot}, R=45.6 R_{\odot}, T_{\text {eff }}=5726 \mathrm{~K}, \log g=1.92$.

Keywords: stellar evolution; stellar pulsation; Cepheids; stars: variable and peculiar
\end{abstract}

*E-mail: fadeyev@inasan.ru 


\section{INTRODUCTION}

According to the General catalogue of variable stars (Samus' et al. 2017) the pulsating variable V1033 Cyg with period of light variations $\Pi=4.9375$ day belongs to $\delta$ Cep type stars. The most remarkable feature of V1033 Cyg is the enormously high rate of period increase. The study of the secular period change shows that the $O-C$ diagram spanning last 117 yr can be described with a high accuracy by the quadratic dependence, whereas the period change rate is $\dot{\Pi}=18.19 \mathrm{~s} /$ yr with a r.m.s. error $\sigma \approx 0.08 \mathrm{~s} / \mathrm{yr}$ (Berdnikov et al. 2019). This value of $\dot{\Pi}$ is several dozen times larger than that of most core helium burning Cepheids (Fadeyev 2013, 2014). V1033 Cyg seems to be in the relatively short evolutionary stage of gravitational contraction of the helium core during which the star crosses the Hertzsprung-Russel (HR) diagram in the thermal time scale. This assumption is confirmed by detection of ${ }^{7} \mathrm{Li}$ lines indicating that the star is in the stage preceding the red giant (Luck and Lambert 2011). Thus, V1033 Cyg seems to be the second Cepheid known as a post-main sequence star crossing the instability strip for the first time. Earlier the evolutionary status of the Cepheid at the first crossing has been established for $\alpha$ UMi (Turner et al. 2005; Fadeyev 2015a).

Owing to the small error of the observational estimate of $\dot{\Pi}$ the Cepheid V1033 becomes a very appropriate object for determination of its fundamental parameters (i.e. the stellar age $t_{\mathrm{ev}}$, the mass $M$, the luminosity $L$ and the radius $R$ ) by means of comparison of theoretically computed $\Pi$ and $\dot{\Pi}$ with observations. The method is based on consistent calculations of stellar evolution and nonlinear stellar pulsations where selected models of evolutionary sequences are used as initial conditions in solution of the Cauchy problem for the equations of radiation hydrodynamics and time-dependent convection describing radial stellar pulsations. Earlier this method was employed for determination of the fundamental parameters of the Cepheids $\alpha$ UMi (Fadeyev 2015a) and SZ Tau (2015b) as well as of groups of long-period (Fadeyev 2018) and short-period Cepheids (Fadeyev 2020). Efficiency of the method is confirmed by a good agreement (within several percent) of the results of theoretical computations with BaadeWesselink radius measurements for long-period Cepheids RS Pup, GY Sge and SV Vul.

The goal of the present study is to confirm the evolutionary status of V1033 Cyg as a Cepheid crossing the instability strip for the first time as well as to determine its fundamental parameters by methods of the theory of stellar evolution and the theory of stellar pulsation. Evolutionary computations were done with the program MESA version 12115 (Paxton et al. 2018). Calculations of evolutionary sequences and nonlinear stellar pulsations are discussed in our preceding paper (Fadeyev 2020). Initial conditions necessary for solution of the equations of hydrodynamics were obtained from selected models of evolutionary sequences with initial masses $5.7 M_{\odot} \leq M \leq 7.4 M_{\odot}$ and initial fractional abundance of helium $Y=0.28$. Bearing 
in mind uncertainties in Cepheid metallicities due to the existence of the galactic abundance gradient (Luck and Lambert 2011; Luck et al. 2011; Lemasle et al. 2018) the stellar evolution and the stellar pulsation calculations were done for the standard metallicity $Z=0.02$ as well as for $Z=0.018$ and $Z=0.022$.

\section{RESULTS OF CALCULATIONS}

Evolutionary sequences were computed from the initial main sequence up to the stage preceding the evolution along the red giant branch on the HR diagram. Effects of mass loss due to the stellar wind in main sequence stars with masses $M \leq 7.4 M_{\odot}$ remain negligible since the star loses less than $10^{-4}$ of its total mass. Moreover, in the outer layers with the temperature $T \lesssim 5 \times 10^{6} \mathrm{~K}$ and the radius $r \geq 0.1 R$, where pulsation motions are perceptible, abundances of chemical elements do not undergo evolutionary changes. Therefore, without significant loss of accuracy we can assume that the masses of Cepheid hydrodynamic models as well as abundances of chemical elements are the same as on the initial main sequence.

During the first crossing of the instability strip the pulsation period monotonically increases, evolution of stars with masses $M \leq 7 M_{\odot}$ being accompanied by switch of oscillations from the first overtone to the fundamental mode. To determine with sufficient accuracy the star age corresponding to pulsation mode switching we computed for each evolutionary sequence $\approx 15$ hydrodynamic models uniformly distributed with respect to $t_{\mathrm{ev}}$ between edges of the instability strip. According to approach employed in our preceding study (Fadeyev 2019) we assumed that the star age at the pulsation mode switch is the mean value of the ages of two adjacent hydrodynamic models pulsating in different modes. Within the interval with continuous change of the pulsation period the dependence $\Pi\left(t_{\mathrm{ev}}\right)$ was calculated using a least-squares polynomial fit. For polynomials of the third order the r.m.s. error of approximation does not exceed 0.1 percent.

Results of consistent stellar evolution and nonlinear stellar pulsation computations are illustrated in Fig. 1 where the plots of evolutionary variations of $\Pi$ and $\dot{\Pi}$ are shown for Cepheids with metal abundance $Z=0.02$. For the sake of graphical convenience the horizontal coordinate is set to zero when the star of the age $t_{\mathrm{ev}, \mathrm{b}}$ crosses the blue edge of the instability strip. The final point of each plot represents decaying pulsations at the red edge of the instability strip. The duration of the first crossing of the instability strip lasts between $\approx 5.3 \times 10^{3} \mathrm{yr}$ for $M=7.4 M_{\odot}$ and $\approx 1.8 \times 10^{4} \mathrm{yr}$ for $M=5.7 M_{\odot}$. The horizontal dashed lines in Figs. 1 1 a and $1 \mathrm{~b}$ are shown for comparison of the results of computations with observations and correspond to the period $\Pi=4.9403 \mathrm{yr}$ and the rate of period change $\dot{\Pi}=18.19 \mathrm{~s} / \mathrm{yr}$ (Berdnikov 2019).

The abrupt period change shown in plots by dotted lines is due to pulsation mode switching from the first overtone to the fundamental mode. As can be seen in Fig. 11a, the pulsation mode 
switching significantly constrains the mass interval of stars pulsating with period $\Pi \approx 4.94$ day. In particular, this condition is fulfilled for the fundamental mode pulsators of evolutionary sequences with masses $5.9 M_{\odot} \lesssim M \lesssim 6.1 M_{\odot}$.

In Cepheids at the first crossing of the instability strip the periods of the fundamental mode $\Pi_{0}$ and of the first overtone $\Pi_{1}$ at the point of pulsation mode switching monotonically increase with increasing stellar mass. For evolutionary sequences with initial masses ranged within $5.7 M_{\odot} \leq M \leq 7.0 M_{\odot}$ the increase of the period is described by relations

$$
\begin{aligned}
& \Pi_{0}=-6.962+1.926 \mathrm{M} / M_{\odot}, \\
& \Pi_{1}=-4.413+1.258 \mathrm{M} / M_{\odot},
\end{aligned}
$$

where $\Pi_{0}$ and $\Pi_{1}$ are expressed in days. Dependencies (1) and (2) represent the linear approximation of $\Pi_{0}$ and $\Pi_{1}$ at the points of mode switching (see Fig. 2) and were determined from hydrodynamic computations.

Attempts to compute the hydrodynamic model of the Cepheid V1033 Cyg pulsating in the first overtone have failed. This is due to the two following reasons. First, radial oscillations in the first overtone are excited in stars with masses $M \leq 7 M_{\odot}$ and the upper limit of the period is $\Pi \approx 4.4$ day. Second, if we succeed to compute the hydrodynamic model for the first overtone pulsator with mass $M>7 M_{\odot}$ (for example, via variations of stellar chemical composition) then, as can be seen in Fig. 1 b, the growth of the period change rate with increasing stellar mass inevitably leads to contradiction with observations.

To obtain more accurate estimates of the fundamental parameters of V1033 Cyg determined from hydrodynamic models of the fundamental mode pulsators we computed additional evolutionary sequences of the stars with masses $M=5.9 M_{\odot}, 6.1 M_{\odot}$ and $6.3 M_{\odot}$ for metallicities $Z=0.018$ and $Z=0.022$. Results of calculations are presented in the diagram period-rate of period change shown in Fig. 3. The plots demonstrate evolutionary changes of the fundamental mode period and of the rate of period increase between the point of mode switching and the red edge of the instability strip. As can be seen in Fig. 3 , increase of metallicity $Z$ for a fixed value of the stellar mass $M$ is accompanied by displacement of the plot to smaller $\dot{\Pi}$.

As a criterion of agreement between the theory and observations we will use the quantity $\delta=\left|\dot{\Pi} / \dot{\Pi}_{\star}-1\right|$, where $\dot{\Pi}_{\star}=18.19$ is the observational estimate of the period change rate and $\dot{\Pi}$ is the theoretical estimate corresponding to the pulsation period of the hydrodynamic model $\Pi=4.9403$ day. The best agreement with observations $\left(\delta \lesssim 10^{-2}\right)$ in the diagram period rate of period change is obtained for the evolutionary sequence $Z=0.022, M=6.3 M_{\odot}$. The difference between theoretical and observational estimates of $\dot{\Pi}$ increases with decreasing $Z$ and is $\delta \approx 0.05$ for $Z=0.020$ and $\delta \approx 0.1$ for $Z=0.018$.

Fundamental parameters of the Cepheid V1033 Cyg are listed in Table 1. The mass $M$ and metallicity $Z$ given in the first and in the second columns correspond to the evolutionary 
sequence with minimum deviation $\delta$. The evolutionary time $t_{\mathrm{ev}}$ in the third column is the age of the hydrodynamic model pulsating with period 4.9403 day. The luminosity $L$, the radius $R$, the effective temperature $T_{\text {eff }}$ and the surface gravity $g$ were evaluated by interpolation of the results of evolutionary calculations for the star age $t_{\mathrm{ev}}$. Theoretical period change rates $\dot{\Pi}$ given in the last column were calculated using the approximate nonlinear dependences $\dot{\Pi}\left(t_{\mathrm{ev}}\right)$.

\section{CONCLUSIONS}

Results of computations presented in this paper allow us to confidently conclude that the Cepheid V1033 Cyg is the post-main sequence star in the evolution stage of gravitational contraction of the helium core. The star crosses the instability strip for the first time and moves across the HR diagram to the region of red giants. Analysis of our hydrodynamic models confirms assumption on fundamental mode pulsations of V1033 Cyg proposed by Udovichenko et al. (2019) on the basis of photometric measurements. Owing to insignificant role of mass loss in main sequence evolution of intermediate-mass stars we obtained the fairly accurate theoretical estimates of the fundamental parameters. Due to existing uncertainties in metallicities $(0.018 \leq Z \leq 0.022)$ the theoretical estimates of stellar mass range within $5.9 M_{\odot} \lesssim M \lesssim 6.3 M_{\odot}$. It should also be noted that our theoretical estimates of surface gravity $(1.91 \lesssim \log g \lesssim 1.92)$ are in satisfactory agreement with $\log g=2.10$ obtained for V1033 Cyg from calculations of stellar atmosphere models (Martin et al. 2015). 


\section{REFERENCES}

1. L.N. Berdnikov, E.N. Pastukhova, V.V. Kovtyukh, B. Lemasle, A.Yu. Kniazev, I.A. Usenko, G. Bono, E. Grebel, G. Hajdu, S.V. Zhuiko, S.N. Udovichenko, and L.E. Keir, Astron. Lett. 45, 227 (2019).

2. Yu.A. Fadeyev, Astron. Lett. 39, 746 (2013).

3. Yu.A. Fadeyev, Astron.Lett. 40, 301 (2014).

4. Yu.A. Fadeyev, MNRAS 449, 1011 (2015a).

5. Yu.A. Fadeyev, Astron. Lett. 41, 640 (2015b).

6. Yu.A. Fadeyev, Astron. Lett. 44, 782 (2018).

7. Yu.A. Fadeyev, Astron. Lett. 45, 353 (2019).

8. Yu.A. Fadeyev, Astron. Lett. 46, in press (2020).

9. B. Lemasle, G. Hajdu, V. Kovtyukh, L. Inno, E.K. Grebel, M. Catelan, G. Bono, P. François, A. Kniazev, R. da Silva, and J. Storm, Astron. Astrophys. 618, A160 (2018).

10. R.E. Luck and D.L. Lambert, Astron. J. 142, 136 (2011).

11. R.E. Luck, S.M. Andrievsky, V.V. Kovtyukh, W. Gieren, W. and D. Graczyk, Astron. J. 142, 51 (2011).

12. R.P. Martin, S.M. Andrievsky, V.V. Kovtyukh, S.A. Korotin, I.A. Yegorova, I. Saviane, MNRAS 449, 4071 (2015).

13. B. Paxton, J. Schwab, E.B. Bauer, L. Bildsten, S. Blinnikov, P. Duffell, R. Farmer, J.A. Goldberg, P. Marchant, E. Sorokina, A. Thoul, R.H.D. Townsend, and F.X. Timmes, Astropys. J. Suppl. Ser. 234, 34 (2018).

14. N.N. Samus', E.V. Kazarovets, O.V. Durlevich, N.N. Kireeva, and E.N. Pastukhova, Astron. Rep. 61, 80 (2017).

15. D.G. Turner, J. Savoy, J. Derrah, M. Abdel-Sabour Abdel-Latif, and L.N. Berdnikov, Publ. Astron. Soc. Pacific 117, 207 (2005).

16. S.N. Udovichenko, V.V. Kovtyukh, and L.E. Keir, Odessa Astron. Publ. 32, 83 (2019). 
Table 1. Fundamental parameters of the Cepheid V1033 Cyg

\begin{tabular}{cccccccc}
\hline$M / M_{\odot}$ & $Z$ & $t_{\text {ev }}, 10^{6}$ лет & $L / L_{\odot}$ & $R / R_{\odot}$ & $T_{\text {eff }}, \mathrm{K}$ & $\log g$ & $\dot{\Pi}, \mathrm{s} / \mathrm{yr}$ \\
\hline 5.9 & 0.018 & 65.645 & 1648 & 44.6 & 5510 & 1.910 & 16.57 \\
6.1 & 0.020 & 62.005 & 1802 & 45.1 & 5604 & 1.915 & 17.34 \\
6.3 & 0.022 & 58.431 & 2009 & 45.6 & 5726 & 1.919 & 18.36 \\
\hline
\end{tabular}




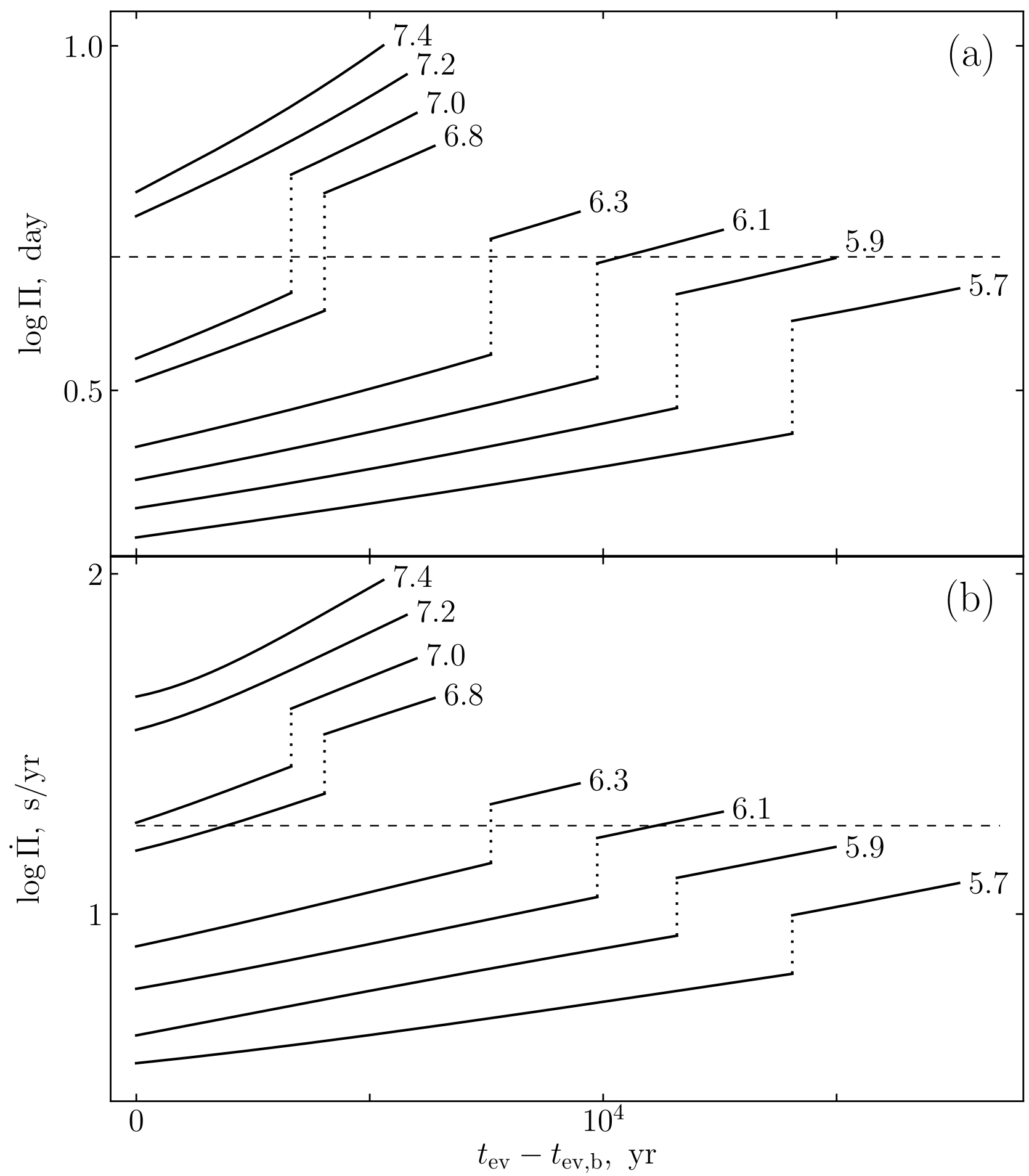

Figure 1. Temporal variations of the pulsation period (a) and of the rate of period change (b) in radially pulsating Cepheids with $Z=0.02$ during the first crossing of the instability strip. The stellar masses $M$ are indicated at the tracks. Dotted lines correspond to pulsation mode switching. Horizontal dashed lines show the observational estimates of the period $\Pi=4.9403$ day and of the period change rate $\dot{\Pi}=18.19 \mathrm{~s} /$ yr (Berdnikov et al. 2019). 


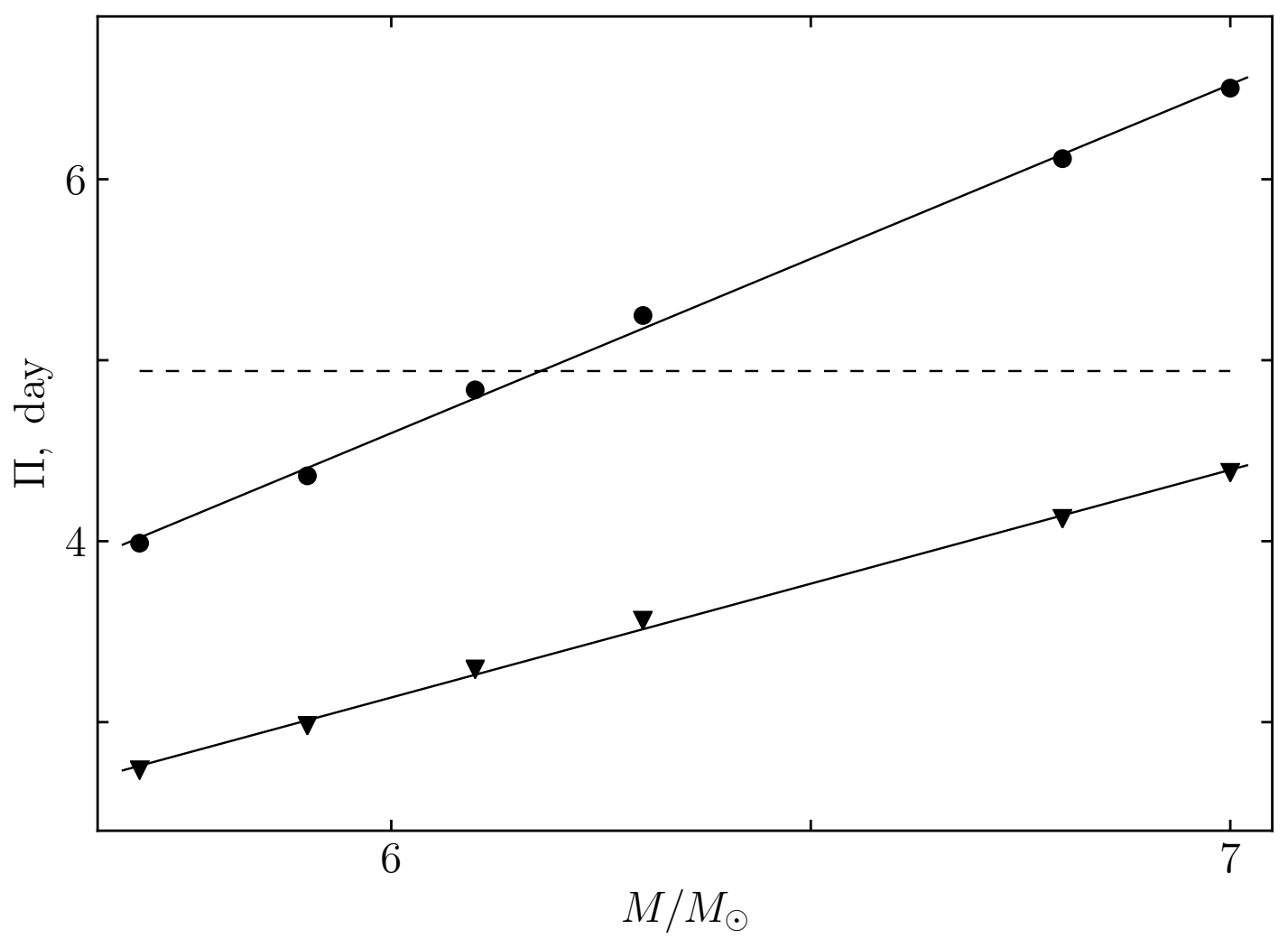

Figure 2. Periods of the fundamental mode $\Pi_{0}$ (filled circles) and of the first overtone $\Pi_{1}$ (filled triangles) at the point of mode switching as a function of stellar mass $M$ for evolutionary sequences with metallicity $Z=0.02$. The horizontal dashed line shows the period $\Pi=4.9403$ day. 


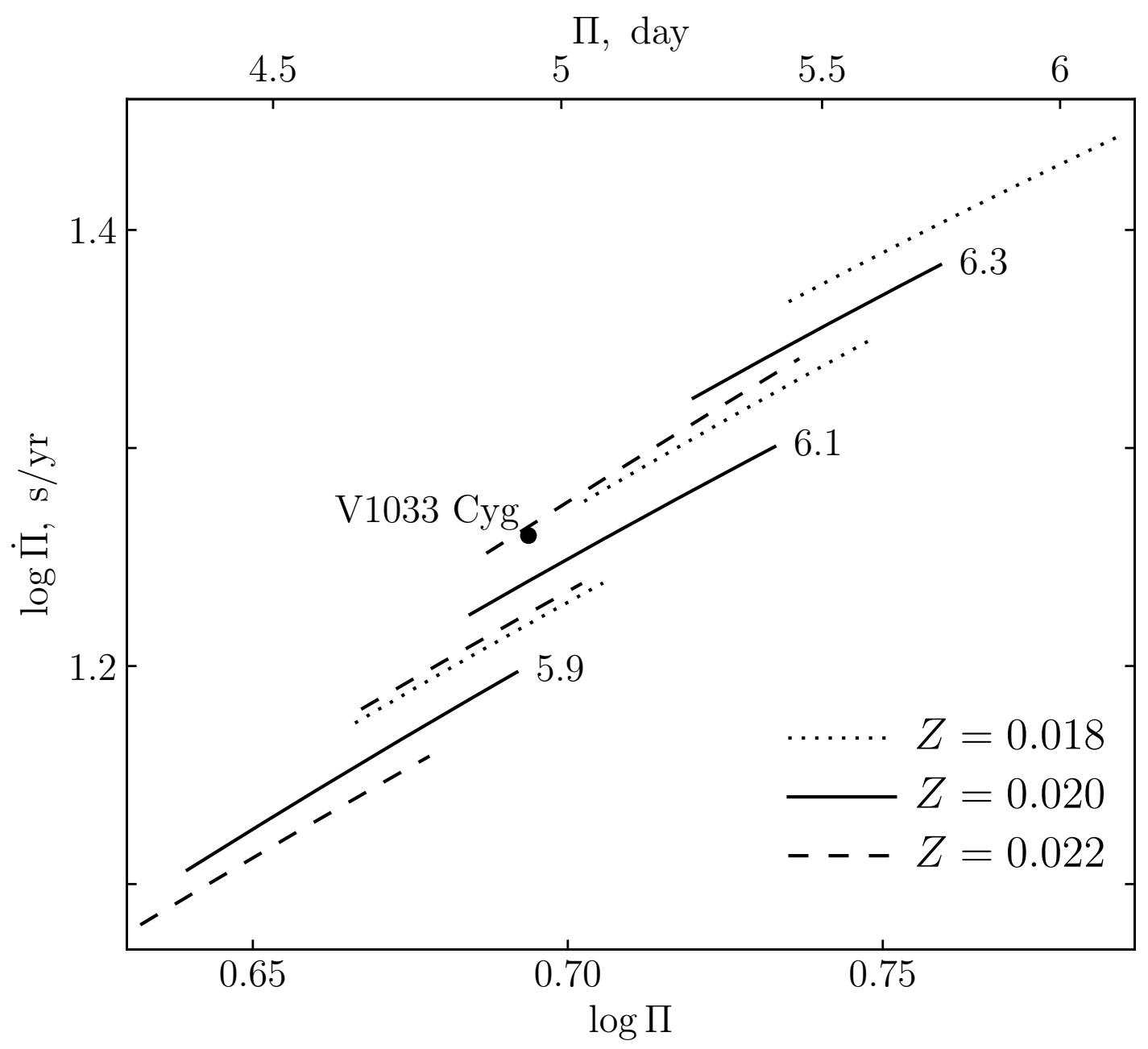

Figure 3. The rate of period change $\dot{\Pi}$ as a function of period $\Pi$ for Cepheid models pulsating in the fundamental mode under different assumptions on metallicuty $Z$. The numbers at the plots indicate the stellar mass $M$. The filled circle shows the Cepheid V1033 Cyg (Berdnikov et al.2019). 